\title{
Coded Multipulse Pulse-Position Modulation for Free-Space Optical Communications
}

\author{
Trung Thanh Nguyen, Student Member, IEEE, and Lutz Lampe, Senior Member, IEEE
}

\begin{abstract}
In this letter, we address two questions concerning the application of multipulse pulse-position modulation (MPPM) for free-space optical (FSO) communications: (1) under what condition would MPPM offer a notable advantage over conventional pulse-position modulation (PPM), and (2) what practical coding scheme should be used to realize that advantage. Focusing on bandwidth-efficient FSO transmission, we find decimated MPPM constellations which are suitable for combining with binary codes and offer significant gains in terms of constellationconstrained capacity over PPM under simultaneous peak-power, average-power, and bandwidth constraints. We then consider labeling design for the popular bit-interleaved coded modulation (BICM) and devise a new multilevel coding (MLC) architecture for MPPM, which we refer to as reduced-layer MLC (RLMLC). Considering the Poisson FSO channel model as a relevant example, we provide simulative evidence that RL-MLC MPPM with off-the-shelf low-density parity-check codes can outperform any PPM scheme under the same transmission constraints.
\end{abstract}

Index Terms-Multipulse pulse-position modulation (MPPM), multilevel coding (MLC), bit-interleaved coded modulation (BICM), free-space optical (FSO) transmission.

\section{INTRODUCTION}

On-off keying (OOK) and pulse-position modulation (PPM) are two popular modulation formats for free-space optical (FSO) communications [1]. OOK is more bandwidth-efficient than PPM, but it has several drawbacks such as the need to select an appropriate decision threshold at the receiver and more complex synchronization due to possible long sequences of zeros or ones [2]. The biggest disadvantage of PPM is the rapid decline of bandwidth efficiency with increasing power efficiency afforded by larger constellation sizes. For these reasons, multipulse pulse-position modulation (MPPM) has been proposed as an alternative modulation format to PPM and OOK [3]. MPPM is a generalization of PPM in that $w>1$ pulses are transmitted during a symbol period of $n$ slots, which enables higher bandwidth efficiency than PPM.

In this letter, we consider the combination of error-control coding (ECC) with MPPM to achieve power- and bandwidthefficient FSO transmission. In particular, we are interested in the application of binary ECC schemes for which a large class of powerful codes have been developed in the past fifty years. We note that when MPPM is employed, the original

Manuscript received January 22, 2009; revised October 1, 2009. This work was supported by the National Sciences and Engineering Research Council (NSERC) of Canada. Part of this work was presented at the International Symposium on Communication Systems, Networks, and Digital Signal Processing (CSNDSP), Graz, Austria, July 2008.

The authors are with the Deptartment of Electrical and Computer Engineering, University of British Columbia, Vancouver, Canada (e-mail: trungn@ece.ubc.ca, Lampe@ece.ubc.ca). constellation size is not a power of two, and thus the bitto-symbol mapping is complicated. A common-sense solution is to decimate the constellation to the nearest power-of-two size. Since this in turn reduces bandwidth efficiency, and since MPPM symbols are not orthogonal and thus require higher symbol energy for the same uncoded symbol error rate than PPM to start with, (at least) two questions immediately arise. Firstly, under what circumstances would MPPM indeed offer notable throughput or power efficiency gains over PPM? Secondly, how can those gains be realized by practical ECC schemes?

To address the first question, MPPM and PPM have been compared based on different criteria and under various transmission conditions, cf. e.g. [3], [4], [5], [6], [2], [7], [8], [9]. It has been shown that although MPPM can potentially be two times more bandwidth efficient than PPM with the same power efficiency [3], considerable throughput gains are only present for high signal power and duty cycle $w / n$ [4], [9]. In terms of error-rate performance, it was recognized that no PPM or MPPM constellation is universally superior to all the others, and that different modulation formats are preferable under different transmission constraints, such as peak- or average-power or bandwidth constraints [2]. Hence, before designing specific ECC schemes for MPPM, a careful selection of appropriate MPPM constellations is needed. As for the second question, we note that several coding schemes have been studied for MPPM. Analytical results for ReedSolomon coded MPPM were given in [4], [5], [10], [6]. Sato et al. [6] also analyzed the performance of convolutional coded 2-pulse MPPM and investigated the effect of imperfect slot synchronization on the error rate performance. The combination of trellis-coded modulation (TCM) with 2-pulse MPPM was outlined in [2]. A more detailed description of TCM with 2-pulse MPPM, including asymptotic coding gain calculation, was given in [11]. Serially concatenated TCM was also considered for 2-level 2-pulse MPPM (each of the two pulses can take two values) in [12]. It should be noted that none of these ECC schemes is capacity-approaching. Hence a significant performance gain is left to be realized. Furthermore, most authors have considered only 2-pulse MPPM, probably for ease of analysis.

In this letter, we revisit both of the above questions concerning the application of coded MPPM. Firstly, in Section III, we compare MPPM with PPM in terms of achievable data rate under simultaneous peak power, average power, and bandwidth constraints. A similar comparison has been performed by Hamkins and Moision [9]. However, they considered full-size MPPM and mostly focused on low duty-cycle modulations for 
deep-space communication, where MPPM's throughput gains are very limited. Our comparison here focuses on high dutycycle and thus bandwidth-efficient MPPM with decimated constellations suitable for coded transmission. Secondly, in Section IV, we investigate the application of binary ECC to MPPM using such decimated MPPM constellations. In particular, we design bit-interleaved coded modulation (BICM) [13] and multilevel coding (MLC) with multistage decoding (MSD) schemes [14] for the problem at hand. In the case of BICM, this mainly concerns the labeling of decimated MPPM constellations. For MLC-MSD, we propose an architecture with a reduced number of encoder-decoder pairs ${ }^{1}$ compared to the original MLC-MSD, which we refer to as reducedlayer MLC (RL-MLC). Using constellation-constrained capacity as the pertinent figure of merit, we find that BICM is only an appropriate ECC scheme for MPPM at relatively high signal-to-noise power ratio (SNR), whereas RL-MLC MPPM outperforms PPM over a wide range of SNR. Bit-errorrate (BER) simulation results using off-the-shelf low-density parity-check (LDPC) codes confirm the superiority of RLMLC MPPM over any coded PPM scheme for the example of FSO transmission over Poisson channels with bandwidth efficiency of $0.45 \mathrm{bit} / \mathrm{slot}$. Finally, we summarize the main results in Section V.

\section{MPPM TRANSMISSION AND CONSTELlation-CONSTRAINED CAPACITY}

In this section, we briefly review MPPM transmission and introduce the constellation-constrained channel capacity, which will be used as the pertinent performance measure in subsequent sections.

MPPM transmission uses intensity-modulation with direct detection (IM/DD), cf. e.g. [1]. Each $(n, w)$-MPPM symbol is represented by a vector $\boldsymbol{x}=\left[x_{1} \ldots x_{n}\right]$ of $n$ binary elements, each of which is transmitted in one slot, and $1<w \leq\lfloor n / 2\rfloor$ elements are ones ("on") and the others are zeros ("off"). The set $\mathcal{U}$ of all distinct $(n, w)$-MPPM symbols has size $M_{\max }=$ $\left(\begin{array}{l}n \\ w\end{array}\right)$, which is never a power of two. Since constellations of sizes $M=2^{m}, m \in \mathbb{Z}$, are preferred for coded transmission, we will use only a decimated constellation $\mathcal{X} \subset \mathcal{U}$ of $M<M_{\max }$ distinct symbols. While there are different IM/DD channel models, suitable for different regimes of operation [1], they are all assumed to be memoryless. We thus can write the average mutual information for a given MPPM constellation $\mathcal{X}$, i.e., constellation-constrained capacity, as

$$
C(\mathcal{X})=-\frac{1}{n} \mathcal{E}\left\{\log \frac{1}{M} \sum_{\boldsymbol{z} \in \mathcal{X}} \prod_{i=1}^{n} \frac{p\left(y_{i} \mid z_{i}\right)}{p\left(y_{i} \mid x_{i}\right)}\right\} \quad[\text { bit/slot }],
$$

where $\boldsymbol{y}=\left[y_{1} \ldots y_{n}\right]$ denotes the received symbol, $p\left(y_{i} \mid x_{i}\right)$ is the conditioned output probability density function (pdf), and $\mathcal{E}\{\cdot\}$ is the expectation operator. As usual, in (1) we have assumed that mapping plus ECC results in equiprobable MPPM symbols. To make matters concrete when presenting numerical results, we apply the very popular discrete-time

\footnotetext{
${ }^{1}$ In the following, we refer to an encoder-decoder pair as a "layer" of the coded systems.
}

Poisson channel model, for which received signal and background radiation intensity are modeled as i.i.d. Poisson random variables with mean $\lambda_{s}$ and $\lambda_{b}$ respectively [1] and thus

$$
p\left(y_{i} \mid x_{i}\right)=\frac{\left(\lambda_{s} x_{i}+\lambda_{b}\right)^{y_{i}}}{y_{i} !} \exp \left[-\left(\lambda_{s} x_{i}+\lambda_{b}\right)\right] .
$$

We would like to emphasize, however, that the proposed methodology is independent of the specific FSO channel model. Finally, we note that the coherence time of random signal intensity fluctuation due to scintillation is assumed much larger than the time horizon for coding and thus only reflects in a change of the SNR, which we define as $\left(w \lambda_{s}\right) /\left(n \lambda_{b}\right)$.

\section{Constellation Selection}

In this section we turn to the problem of selecting "good" MPPM constellations. Our approach consists of two steps. First, we determine parameters $(n, w, M)$ such that MPPM transmission with a corresponding set $\mathcal{X}$ potentially offers throughput gains compared to PPM under the same transmission constraints. Then, we obtain the set $\mathcal{X}$ from decimation of the full-size MPPM set $\mathcal{U}$ such that $C(\mathcal{X})$ is maximized.

1) Parameter Selection: We compare MPPM constellations based on the maximal throughput $\tau=\log _{2}(M) / n$ bit/slot under simultaneous peak power, average power, and bandwidth constraints. Peak and average power constraints require identical duty cycles $1 / \rho=w / n$, where $\rho$ is the peak-toaverage power ratio (PAPR), and the bandwidth constraint is accounted for by measuring throughput in bits per slot. The "OOK bound" $\tau=\mathcal{H}(1 / \rho)$, where $\mathcal{H}(\cdot)$ is the binary entropy function, upper bounds the MPPM throughput for given PAPR $\rho$ [4]. Furthermore, an $n$-slot PPM constellation has $\rho=n$ and $\tau=(\log \rho) / \rho$. Figure 1 shows the power-bandwidth efficiency for MPPM constellations with $M \leq 256$ and $\tau \geq(\log \rho) / \rho$. There are 89 MPPM constellations satisfying these conditions. Each set $(n, w, M)$ is presented as a point in the figure, and several $(n, w, M)$ sets may take the same point. Included are the upper "OOK bound" $\tau=\mathcal{H}(1 / \rho)$ and the lower "PPM bound" $\tau=(\log \rho) / \rho$. With the help of this figure, we are able to locate the parameters $(n, w, M)$ of MPPM constellations that offer both high power and bandwidth efficiencies. For example, if we want a transmission which requires $\rho$ to be around 4.0 we could select one of the three constellations where $(n, w, M)$ equals $(11,3,128),(13,3,256)$, or $(12,3,128)$, for which $M_{\max }=\left(\begin{array}{c}n \\ w\end{array}\right)=165,286$, and 220 , respectively.

2) Capacity-Maximizing Subset Selection: Having determined the MPPM constellation parameters, we need to decide which $M$ out of the $M_{\max }$ symbols are to be used. Selection of subsets based on symbol-error rate or avoidance of long sequences of zeros and ones has been considered in [2], [10], [11]. As mentioned above, here we use the constellationconstrained channel capacity $C(\mathcal{X})$ as the relevant criterion. Therefore, we formulate subset selection as a combinatorial optimization $(\mathrm{CO})$ problem:

$$
\begin{aligned}
& \text { Maximize: } C(\mathcal{X}) \\
& \text { subject to: } \mathcal{X} \subset \mathcal{U} \text { and }|\mathcal{X}|=M
\end{aligned}
$$

Since the solution of this $\mathrm{CO}$ problem with $C(\mathcal{X})$ from (1) seems to require total enumeration, we aim at a possibly 


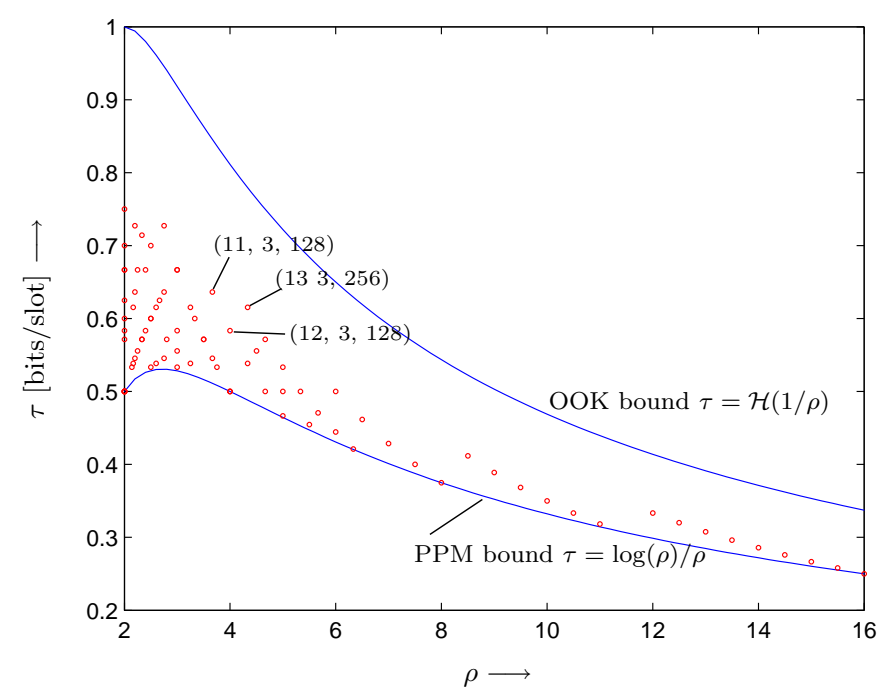

Fig. 1. Power-bandwidth efficiency of MPPM. Parameter sets are triplets $(n, w, M)$.

suboptimal solution using $\mathrm{CO}$ search strategies such as random search, greedy ascent, and simulated annealing. We refer readers to [15] for more details, including pseudo-code for the search algorithms. As an example, Figure 2 shows the constellation-constrained capacity for $\mathcal{U}(12,3,220)$ (line (a)) and the optimized subset $\mathcal{X}(12,3,128)$ found by random search (line (b)) as a function of SNR, where $\lambda_{b}=0.2$ [16]. The numerical $\mathrm{CO}$ search has been performed for SNR = $6.9 \mathrm{~dB}$, at which $C(\mathcal{U})=\log M$, and we have found that a constellation optimized for this SNR value performs well for a wide range of SNRs. For a fair comparison, the capacity for 4-PPM (line (i)) is included, which has the same PAPR of $\rho=4.0$. We observe that the selected $(12,3,128)$ MPPM constellation always outperforms 4-PPM for the entire range of signal power $\lambda_{s}$. The capacity gap is larger at higher SNR, and gradually vanishes at lower SNR values.

\section{CODING FOR MPPM}

Having found a good MPPM constellation $\mathcal{X}$, we now consider the combination of MPPM with coding. Since we deal with multilevel signal constellations, it is natural to apply MLC, which allows us to re-use off-the-shelf binary codes. The main disadvantage of optimal MLC-MSD is the large number $(m=\log M)$ of encoders and decoders, which also entails a fine-tuned code design. For this reason, we first study single-layer MLC, i.e., the popular BICM, for MPPM, and then we propose RL-MLC to maximize performance for a given number of encoder-decoder pairs.

\section{A. BICM for MPPM}

Let us denote the binary label of an MPPM signal point by $\boldsymbol{b}=\left[b^{1} \ldots b^{m}\right]$. Then we can express the constellationconstrained capacity from (1) as [14]

$$
C(\mathcal{X})=\sum_{i=1}^{m} C^{i}
$$

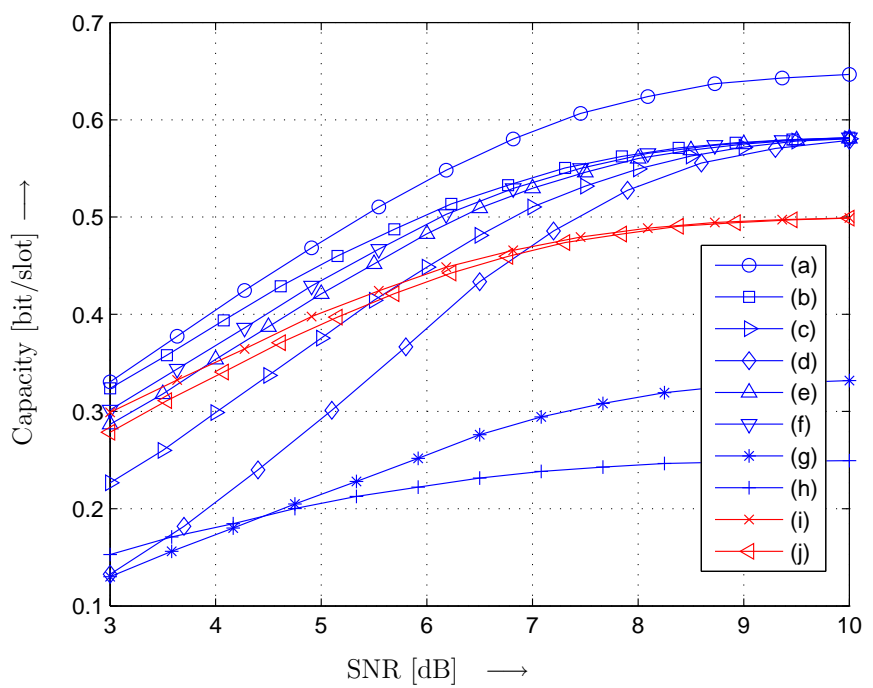

Fig. 2. Capacities of Poisson channel with average background radiation $\lambda_{b}=0.2$ and different constellations and schemes: (a) Full 220-symbol MPPM with $(n, w)=(12,3)$; (b) Selected subset $(n, w, M)=(12,3,128)$ by a random search; (c) BICM with Gray-like labeling obtained with Algorithm 1 (Figure 3); (d) BICM with labeling from a random search; (e) Best 2-layer RL-MLC; (f) Best 3-layer RL-MLC; (g) Layer 1 of the best 2-layer RL-MLC; (h) Layer 2 of the best 2-layer RL-MLC. For comparison, (i) and (j) are the channel capacity and BICM capacity of 4-PPM, which has the same PAPR $\rho$ as $(12,3)$-MPPM.

where

$$
C^{i}=I\left(\boldsymbol{Y} ; B^{i} \mid B^{1}, B^{2}, \ldots, B^{i-1}\right),
$$

and $I(\cdot ; \cdot)$ denotes mutual information and upper case letters $\boldsymbol{Y}$ and $B^{i}$ represent the random variables corresponding to received signal vector $\boldsymbol{y}$ and label $b^{i}$. This rate is achievable by MLC-MSD using $m$ encoder-decoder pairs for transmission over equivalent binary channels with capacities $C^{i}$. BICM can be viewed as MLC with parallel decoding of levels (PDL), and the associated constellation-constrained capacity is given by

$$
C_{\mathrm{BICM}}(\mathcal{X})=\sum_{i=1}^{m} C_{\mathrm{BICM}}^{i}
$$

where

$$
C_{\mathrm{BICM}}^{i}=I\left(\boldsymbol{Y} ; B^{i}\right) \leq C^{i} .
$$

The capacity loss compared to MLC-MSD strongly depends on the labeling of signal points.

Typically, Gray labeling is suggested for use with BICM [13], [17]. In Gray labeling, the labels of nearest-neighbor signal points differ at only one position. For MPPM, where adjacency is measured in terms of Hamming distance between signal vectors, we may not be able to construct a Gray labeling. This is because a necessary condition for the existence of a Gray labeling is that the number of nearest-neighbor signal points to any signal point must not exceed the number of labeling bits. In most relevant cases, MPPM constellations do not satisfy this condition. As an example, for the $(12,3,128)$ constellation found in Section III, each constellation point has between 11 and 19 nearest neighbors, whereas $m=7$. We therefore attempt to find labelings $\mathcal{L}$ that are "as Gray as 
possible." To this end, we consider the $\mathrm{CO}$ problem

Mimimize: $f_{G}(\mathcal{L})=\frac{1}{M} \sum_{i=1}^{M} \frac{\sum_{\boldsymbol{x}_{j} \in \mathcal{N}\left(\boldsymbol{x}_{i}\right)} d_{H}\left(\boldsymbol{b}_{i}, \boldsymbol{b}_{j}\right)}{\left|\mathcal{N}\left(\boldsymbol{x}_{i}\right)\right|}$,

subject to: $\left\{\boldsymbol{b}_{i} \in\{0,1\}^{m} \mid i=1, \ldots, M\right\}$,

where $\mathcal{N}\left(\boldsymbol{x}_{i}\right)$ is the set of the nearest neighbors of $\boldsymbol{x}_{i}, \boldsymbol{b}_{i}$ is the label of $\boldsymbol{x}_{i}$, and $d_{H}\left(\boldsymbol{b}_{i}, \boldsymbol{b}_{j}\right)$ denotes the Hamming distance between labels $\boldsymbol{b}_{i}$ and $\boldsymbol{b}_{j}$. We note that the cost function $f_{G}(\mathcal{L}) \geq 1$ is the average Hamming distance between labels of nearest-neighbor symbols, and $f_{G}(\mathcal{L})=1.0$ iff $\mathcal{L}$ is a Gray labeling. Incidentally, a similar cost function has been applied in [18, Eqs. (4), (5)] for BICM design for radio communication over additive white Gaussian noise (AWGN) channels.

Again, finding the optimal solution of (6) might require the prohibitive total enumeration. We therefore propose a "local search with best improvement and random restart" algorithm, which we refer to as Algorithm 1 in the following and whose pseudo-code is listed in Figure 3. Figure 2 (line (c)) shows the BICM capacity corresponding to the optimized labeling for the $(12,3,128)$ constellation from Section III and $N=1000$ search steps in Algorithm 1. To illustrate the effectiveness of Algorithm 1, we also compare the result with labelings found by a search over 1000 randomly generated labelings (line (d) in Figure 2). We observe that the Gray-like labeling obtained from solving (6) with Algorithm 1 significantly improves the BICM capacity. However, while the capacity associated with 4-PPM BICM (line (j)) is virtually as good as 4-PPM MLC (line (i)), MPPM BICM leaves a notable gap to the MPPM MLC capacity (line (b)) especially at medium-to-low SNR values. We therefore propose an RL-MLC architecture in the next section, which makes use of the BICM-optimized labeling found here, and is able to narrow this gap.

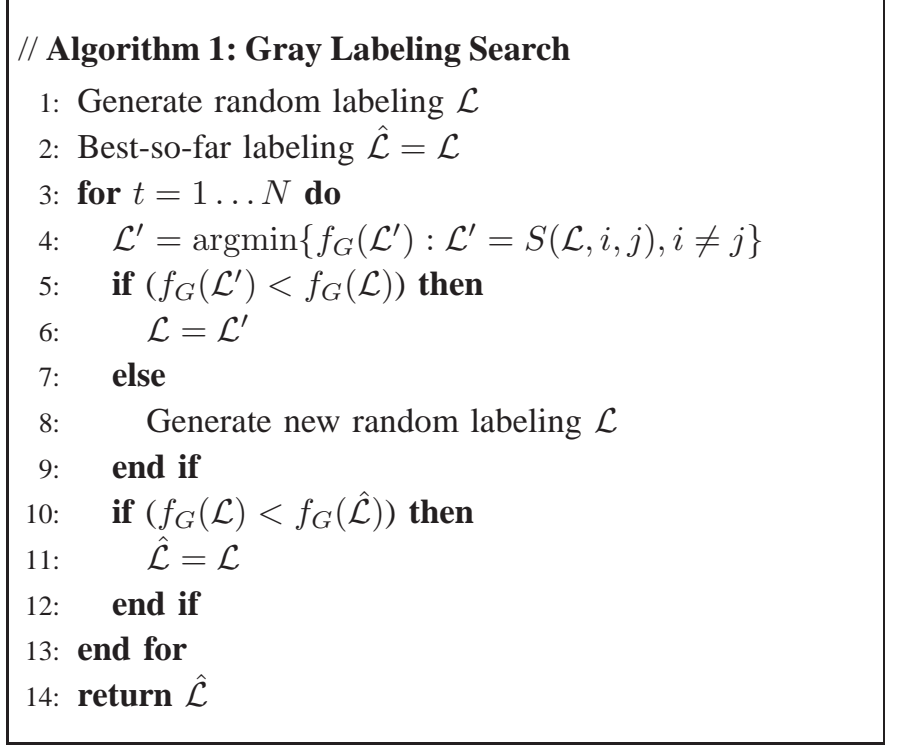

Fig. 3. Pseudo-code for Gray labeling search. $f_{G}(\mathcal{L})$ is the cost function defined in (6) and $S(\mathcal{L}, i, j)$ denotes the label-swapping operation applied on $\mathcal{L}$ such that the labels of the $i$-th and $j$-th symbols are swapped.

\section{B. Reduced-layer MLC for MPPM}

The rationale behind RL-MLC is to reduce the number of coding layers compared to MLC-MSD and as such to tradeoff performance for design and implementation complexity of the coding architecture.

To explain our methodology, consider the MSD architecture for an $(m=3)$-layer constellation in Figure 4 (top). Each line $L_{j, i}$ connecting decoders $D_{i}$ and $D_{j}$ for $j<i$ is denoted a dependency link (DL). When all DLs are eliminated, MSD becomes PDL, which leads to BICM. When only some DLs are eliminated, it may be possible to group a number of levels into layers. Within the same layer, levels can share the same binary encoder and decoder, as in BICM, and we obtain RLMLC. An example of a 3-bit 2-layer RL-MLC configuration is given in Figure 4 (middle and bottom) with the DL $L_{1,2}$ between levels 1 and 2 eliminated. Now instead of three encoder-decoder pairs as in MLC-MSD, only two are needed for RL-MLC. Each RL-MLC configuration has a set $\mathcal{D}$ of remaining DLs. The corresponding constellation-constrained capacity is given by

$$
C_{\mathrm{RL}-\mathrm{MLC}}(\mathcal{X})=\sum_{i=1}^{m} C_{\mathcal{D}}^{i}
$$

where

$$
C_{\mathcal{D}}^{i}=I\left(\boldsymbol{Y} ; B^{i} \mid\left\{B^{j}: L_{j, i} \in \mathcal{D}\right\}\right) .
$$

Since $C_{\mathrm{BICM}}^{i} \leq C_{\mathcal{D}}^{i} \leq C^{i}$, we have $C_{\mathrm{BICM}}(\mathcal{X}) \leq$ $C_{\mathrm{RL}-\mathrm{MLC}}(\mathcal{X}) \leq C(\mathcal{X})$. In order to find the RL-MLC configuration which maximizes (7), we examine the removal of DLs. Given the desired number of layers $\kappa$, the number of possible configurations can be found as $\kappa ! S(m, \kappa)$, where $S(m, \kappa)$ is the Stirling number of the second kind. Hence, full enumeration is possible for small $\kappa$, which is typically the design goal. However, we can expedite the search by precluding weak configurations for which the difference between $C^{i}$ and $C_{\mathrm{RL}-\mathrm{MLC}}^{i}$ after removing a DL exceeds a certain fraction of $C^{i}$. If this is the case, level $i$ should be placed on a high layer so that the DL is retained.

Figure 2 shows $C_{\mathrm{RL}-\mathrm{MLC}}(\mathcal{X})$ for the $(12,3,128)$ constellation from Section III and the best RL-MLC configuration with $\kappa=2$ (line (e)) and $\kappa=3$ (line (f)) layers. Using vector $\boldsymbol{h}$ where the value of $h_{i}$ indicates the layer allocation of level $i$, the best 2- and 3-layer RL-MLC configurations are obtained as $\boldsymbol{h}=\left[\begin{array}{lllllll}1 & 1 & 1 & 1 & 2 & 2 & 2\end{array}\right]$ and $\boldsymbol{h}=\left[\begin{array}{lllllll}1 & 1 & 2 & 2 & 3 & 3 & 3\end{array}\right]$, respectively. We observe that RL-MLC effectively closes the gap between BICM and MLC capacities and thus is an effective way of ECC for MPPM. It is worth pointing out that we have arrived at the RL-MLC schemes via two greedy optimization stages: finding a labeling that maximizes the BICM capacity, and then determining a layer configuration that maximizes the RLMLC capacity for this labeling. Since RL-MLC uses BICM per layer, the first labeling optimization stage is crucial for the overall design. Furthermore, the results in Figure 2 show that there is only very little room for possible improvement by a joint optimization of labeling and layer structure. 

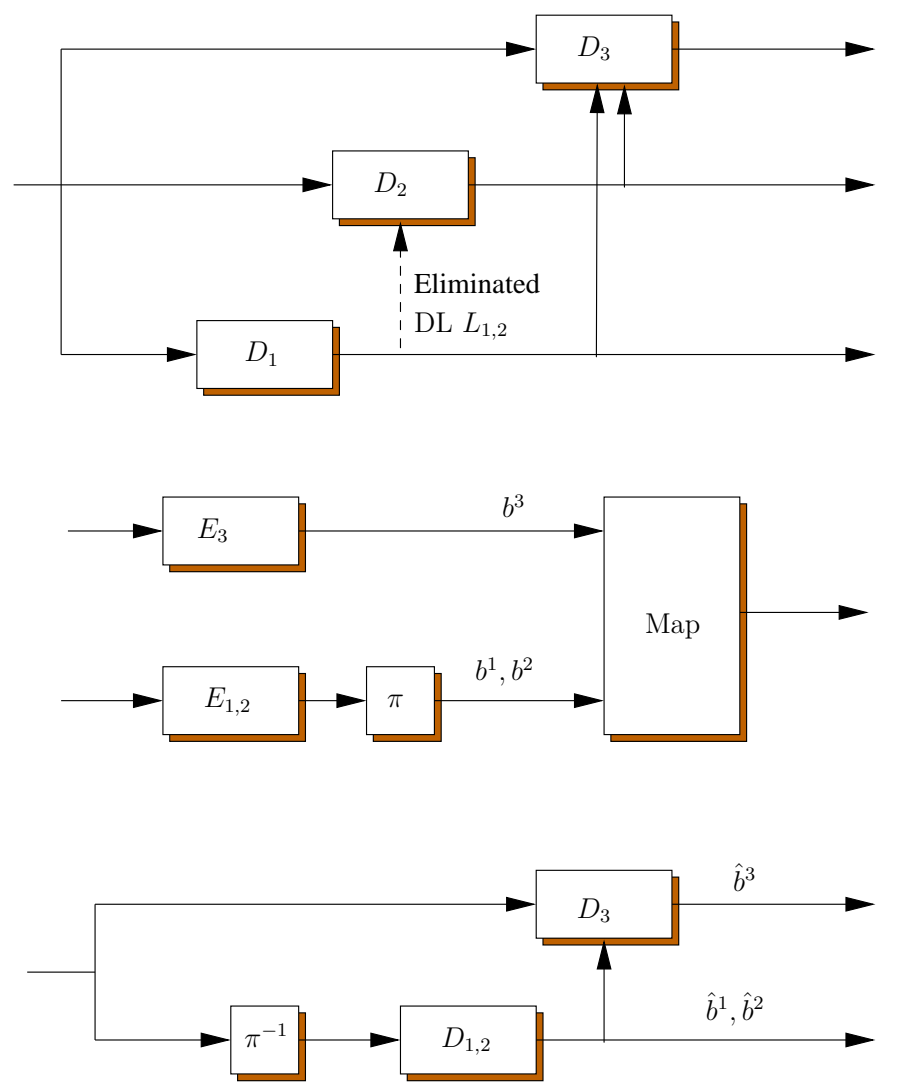

Fig. 4. RL-MLC example. Top: MSD with three binary decoders $D_{i}, i=$ $1,2,3$. DL $L_{1,2}$ is being eliminated. Middle and bottom: corresponding 2 layer RL-MLC transmitter and receiver with two binary encoders $\left(E_{1,2}\right.$ for layer $1, E_{3}$ for layer 2$)$ and two binary decoders $\left(D_{1,2}\right.$ for layer $1, D_{3}$ for layer 2). $\pi$ and $\pi^{-1}$ represent bitwise interleaver and deinterleaver.

\section{Simulation Results Using Binary Codes}

From Figures 1 and 2 we have seen that MPPM is advantageous over PPM at high duty cycles $1 / \rho$, where it offers higher throughput or, through the application of the proposed RLMLC, improved power efficiency for a given bandwidth efficiency. We now provide simulative evidence that the RL-MLC scheme using off-the-shelf binary codes performs close to the capacity limits and thus indeed realizes the MPPM potential. More specifically, we consider the application of LDPC codes for the 2-layer RL-MLC scheme from the previous section. The codes are generated by the progressive edge growth (PEG) algorithm [19] with degree-3 symbol-nodes and almost regular check-nodes. The binary decoders employ the standard sumproduct algorithm in the log domain with a maximum of 200 iterations. We design layer code rates based on the capacity rule [14], i.e. set code rates approximately equal to the layer capacities.

Suppose that we want to transfer a high throughput of 0.45 bit/slot, which is possible at SNR $=5.5 \mathrm{~dB}$, cf. Figure 2, line (e). At this SNR, layer 1 attains approximately 0.24 bit/slot and layer 2 attains approximately $0.21 \mathrm{bit} / \mathrm{slot}$ (cf. Figure 2 lines $(\mathrm{g})$ and $(\mathrm{h})$ ). We use moderate-length codes of sizes 16000 and 12000 for layer 1 and 2 respectively. The code lengths are chosen such that codewords for each layer span 4000 MPPM symbols. The overall code rate of the RL-MLC scheme

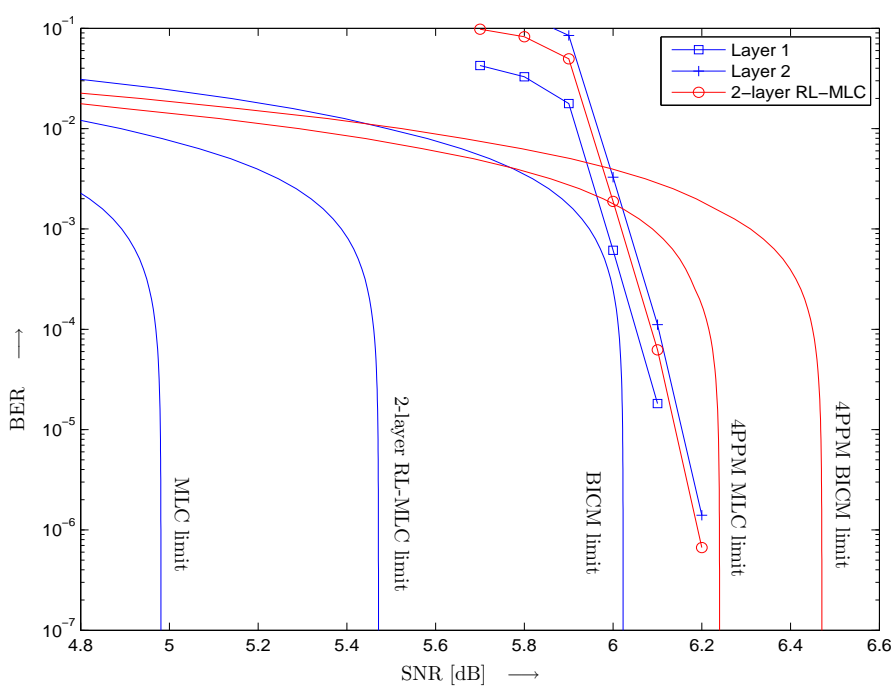

Fig. 5. BER performance for (12,3, 128)-MPPM with 2-layer RL-MLC. Poisson channel with average background radiation $\lambda_{b}=0.2$. Rate-distortion performance limits for MPPM with MLC, 2-layer RL-MLC, BICM, and 4PPM with MLC and BICM are included for comparison.

is 0.77. Figure 5 shows the simulated BER for the overall coding scheme and for the two layers. For a comparison, the rate-distortion capacity limits for MPPM with MLC, 2layer RL-MLC, BICM and 4-PPM with MLC and BICM are also included. We observe that, down to a BER of about $10^{-6}$, 2-layer RL-MLC with LDPC component codes achieves a performance that is within $0.8 \mathrm{~dB}$ of its corresponding capacity limit. Furthermore, its power efficiency (required SNR) is below the capacity limit of 4-PPM. This means, for the targeted high throughput, this RL-MLC MPPM scheme outperforms any coded 4-PPM system and thus corroborates the proposed coded MPPM design.

\section{CONClusion}

In this letter, we have studied coded MPPM FSO transmission. In particular, we have revisited the two fundamental questions of when MPPM is preferable over PPM and how MPPM could be combined with ECC. To this end, we have compared MPPM and PPM FSO transmission under simultaneous limited peak-power, average power, and bandwidth constraints. We then have devised decimation and labeling strategies for the application of ECC to MPPM. It has been found that the popular BICM leaves a considerable gap to channel capacity, which can be bridged by the proposed RLMLC scheme with only few encoder-decoder pairs. Simulation results for an example RL-MLC MPPM design using moderate-length LDPC codes have illustrated that RL-MLC MPPM transmission can outperform any coded PPM scheme under the same transmission constraints.

\section{REFERENCES}

[1] S. J. Dolinar, J. Hamkins, B. E. Moision, and V. A. Vilnrotter, "Optical modulation and coding," in Deep Space Optical Communications, H. Hemmati, Ed. Wiley-Interscience, Apr. 2006.

[2] C. N. Georghiades, "Modulation and coding for throughput-efficient optical systems," IEEE Trans. Inform. Theory, vol. 40, no. 5, Sep. 1994. 
[3] H. Sugiyama and K. Nosu, "MPPM: a method for improving the bandutilization efficiency in optical PPM," J. Lightwave Tech., vol. 7, no. 3, pp. 465-472, Mar. 1989.

[4] M. Takahasi, H. Yashima, I. Sasase, and S. Mori, "Capacity and effects of Reed-Solomon codes on multi-pulse PPM in optical communications," in Proc. IEEE International Communications Conference (ICC), vol. 4, 1990, pp. 1663-1667.

[5] J. M. Budinger, M. J. Vanderaar, P. K. Wagner, S. B. Bibyk, and G. S. Mecherle, "Combinatorial pulse position modulation for power-efficient free-space laser communications," in SPIE Proc., vol. 1866, Jan. 1993, pp. 214-225.

[6] K. Sato, T. Ohtsuki, and I. Sasase, "Performance of coded multi-pulse PPM with imperfect slot synchronization in optical direct-detection channel," in Proc. IEEE International Communications Conference (ICC), vol. 1, 2004, pp. 121-125.

[7] M. K. Simon and V. A. Vilnrotter, "Multi-pulse pulse-position modulation signaling for optical communication with direct detection," IPN Progress Report, vol. 42-155, pp. 1-22, Nov. 2003.

[8] H. Park, "Performance bound on multiple-pulse position modulation," Optical Review, vol. 10, no. 3, pp. 131-132, May 2003.

[9] J. Hamkins and B. Moision, "Multipulse pulse-position modulation on discrete memoryless channels," IPN Progress Report, vol. 42, p. 161, May 2005.

[10] G. E. Atkin and K.-S. L. Fung, "Coded multipulse modulation in optical communication systems," IEEE Trans. Commun., vol. 42, pp. 574-582, Apr. 1994.

[11] H. Park and J. R. Barry, "Trellis-coded multiple-pulse-position modulation for wireless infrared communications," IEEE Trans. Commun., vol. 52, no. 4, pp. 643-651, 2004.

[12] A. S. Alahmari, "Turbo coded pulse position modulation for optical communications," Ph.D. dissertation, Georgia Institute of Technology, 2003.

[13] G. Caire, G. Taricco, and E. Biglieri, "Bit-interleaved coded modulation," IEEE Trans. Inform. Theory, vol. 44, pp. 927-946, May 1998.

[14] U. Wachsmann, R. F. H. Fischer, and J. B. Huber, "Multilevel codes: theoretical concepts and practical design rules," IEEE Trans. Inform. Theory, vol. 45, no. 5, pp. 1367-1391, Jul. 1999.

[15] T. T. Nguyen and L. Lampe, "Capacity-maximized MPPM constellations for free-space optical communications," in Proc. International Symposium on Communication Systems, Networks, and Digital Signal Processing (CSNDSP), Graz, Austria, Jul. 2008, pp. 97-101.

[16] B. Moision and J. Hamkins, "Coded modulation for the deep-space optical channel: Serially concatenated pulse-position modulation," IPN Progress Report, vol. 42, p. 161, May 2005.

[17] C. Stierstorfer and R. F. H. Fischer, "(Gray) Mappings for bit-interleaved coded modulation," in Proc. IEEE Vehicular Technology Conference (VTC-Spring), Dublin, Ireland, Apr. 2007.

[18] S. Le Goff, "Signal constellations for bit-interleaved coded modulation," IEEE Trans. Inform. Theory, vol. 49, pp. 307-313, Jan. 2003.

[19] X.-Y. Hu, E. Eleftheriou, and D. Arnold, "Regular and irregular progressive edge-growth Tanner graphs," IEEE Trans. Inform. Theory, vol. 51, no. 1, pp. 386-398, Jan. 2005. 